\title{
Swine sperm induces neutrophil extracellular traps that entangle sperm and embryos
}

\author{
Zhengkai Wei ${ }^{1}$, Tingting Yu ${ }^{3}$, Jingjing Wang ${ }^{2}$, Chaoqun Wang ${ }^{2}$, Xiao Liu², Zhen Han², \\ Xu Zhang ${ }^{2}$, Yong Zhang ${ }^{2}$, Hongsheng Ouyang ${ }^{3}$ and Zhengtao Yang ${ }^{1}$ \\ ${ }^{1}$ College of Life Sciences and Engineering, Foshan University, Foshan, Guangdong Province, People's Republic of \\ China, ${ }^{2}$ Key Laboratory of Zoonosis, Ministry of Education, College of Veterinary Medicine, Jilin University, \\ Changchun, Jilin Province, People's Republic of China and ${ }^{3}$ Jilin Provincial Key Laboratory of Animal Embryo \\ Engineering, Institute of Zoonosis, College of Animal Sciences, Jilin University, Changchun, Jilin Province, People's \\ Republic of China \\ Correspondence should be addressed to Z Yang; Email: yangzhengtao01@sina.com
}

\begin{abstract}
Sperm motility, fertilization and embryo implantation are several important factors in reproduction. Except healthy state of sperm and embryo themselves, successful pregnancy is closely related to the status of female reproductive tract immune system. Increased immune cells in reproductive tract often leads to low sperm motility and low chance of embryo implantation, but the mechanisms remain not well clarified. The aim of this study is to investigate the direct effects of swine polymorphonuclear neutrophils (PMNs) on sperm or embryo in vitro and then try to clarify the molecular mechanisms undergoing the phenomenon. Swine sperm-triggered neutrophil extracellular traps (NETs) were observed by scanning electron microscopy (SEM). PMNs phagocytosis of sperms was examined by transmission electron microscopy (TEM). Sperm-triggered NETs were quantitated by Pico Green ${ }^{\circledR}$. Vital staining of the interaction between PMNs and embryo were observed by using confocal microscope. It was showed that PMNs were directly activated by sperm in the form of phagocytosis or casting NETs and that sperm-triggered-NETs formation was made up with DNA co-located with citrullinated histone 3 (citH3) and myeloperoxidase (MPO). In addition, the potential mechanism of NETs release was relevant to NADPH oxidase, ERK1/2 or p38 MAPK signaling pathways. Of great interest was that swine embryo was first found entangled in NETs in vitro, but the function and mechanism of this action in vivo fertilization still needed further investigation. In conclusion, this is the first report about swine sperm-induced NETs that entangle sperm and embryo, which might provide an entirely understanding of swine reproductive physiology and immunology.

Reproduction (2020) 160 217-225
\end{abstract}

\section{Introduction}

Artificial insemination has been the principal form for commercial swine breeding on factory farms (Knox 2016, Yeste et al. 2017). As receiving inseminations, polymorphonuclear neutrophils (PMNs) are the main cell type recruited into the swine reproductive tract (Rozeboom et al. 1998, Kaeoket et al. 2003). PMNs play a vital role in early innate immune of the host and can kill microorganisms through phagocytosis, degranulation and neutrophil extracellular traps. In aspects of reproductive immunity, PMNs not only play a critical role in eliminating superfluous spermatozoa and combatting infection via phagocytosis or neutrophil extracellular traps (NETs) (Alghamdi \& Foster 2005, Alghamdi et al. 2009, Hahn et al. 2012, Katila 2012), but are also associated with several stages of reproductive cycle (Wood et al. 2007, Hahn et al. 2012). During natural coitus or artificial insemination, billions of sperm deposited in the reproductive tract also lead to early host innate immune responses and plenty of PMNs release into the uterine immune environments (Fichtner et al. 2020). Once sperm encounter PMNs, PMNs seem capable to eliminate excess sperm by phagocytosis to provide a suitable and favorable microenvironment for embryonic development (Eisenbach 2003). In addition to phagocytosis, PMNs also attack sperm by reactive oxygen species (ROS) synthesis via NOX activation, degranulation and NETs formation (Hahn et al. 2012, Fichtner et al. 2020). However, excess PMNs usually migrate to the reproductive tract under various inflammatory diseases, such as vestibulitis, vaginitis and endometritis, which increase the rate of spermatozoa or embryo exposure to PMNs. In this situation, several mechanisms, including phagocytosis and NETs, may work together to reduce sperm motility or embryo implantation and even result in infertility. However, whether there is such a situation in swine has not been reported. 
Especially, NETs act as a novel mechanism of innate immune system and play a major role in combatting several pathogens, such as bacteria (Brinkmann et al. 2004), virus (Saitoh et al. 2012, Jenne et al. 2013, AgrazCibrian et al. 2017) or parasite (Munoz-Caro et al. 2015a, Wei et al. 2016, 2018a, Yang et al. 2017). These typical NETs consist DNA decorated with citrullinated histone 3 (citH3), myeloperoxidase (MPO), neutrophil elastin (NE) or others. Although the mechanisms of NETs formation remain not very well clarified, reports have showed that several molecular or signaling pathways are involved in NETs release, such as NADPH oxidase, ERK1/2 and p38 MAPK signaling pathways (Munoz-Caro et al. 2015a,b, Wei et al. 2018a). More interestingly, it is reported that NETs formation is a NADPH oxidase-dependent or oxidase-independent process (Douda et al. 2015, Munoz-Caro et al. 2015a, Han et al. 2019). The process of NADPH oxidase-dependent NETs often results in releasing a great deal of mixture consisted with nuclear and cytoplasmic granule including NE, MPO, histone and others (Munoz-Caro et al. 2015a, Han et al. 2019). The other process of NADPH oxidase-independent NETs usually leads to lower production of ERK1/2 and p38 MAPK signaling pathways activation (Douda et al. 2015). Whether these molecular or signaling pathways are also involved in sperm-triggered-NETs release still need to further investigated.

In this study, we aim to first investigate the direct effects of swine PMNs on sperm and then to clarify the molecular mechanisms undergoing the phenomenon. In addition, we also try to assess the effects of swine PMNs on diploid or tetraploid embryo implantation in vitro.

\section{Materials and methods}

\section{Swine PMN isolation}

Blood of healthy swine $(n=3)$ was collected through puncture of the precaval vein and kept in heparinized tubes. PMNs were isolated by using swine PMN isolation $\mathrm{kit}^{\circledR}$ (Tian)in HaoYang Biological Manufacture CO., China). First, preparing separation fluid: $3 \mathrm{~mL}$ regent $\mathrm{A}$ was added in a 15 -mL-centrifuge tube and then $2 \mathrm{~mL}$ regent $C$ was slowly added on regent $A$ (regent $A$ and regent $C$ were provided by swine PMN isolation $\mathrm{kit}^{\circledR}$ ). Secondly, anticoagulation was added on the separation fluid and centrifuged at $650 \mathrm{~g}$ for $20 \mathrm{~min}$. After centrifugation, two circular layers of white cells appear in the centrifuge tube: the upper are mononuclear cells and the lower are polymorphonuclear neutrophils. Thirdly, polymorphonuclear neutrophils were collected, and red blood cells were lysed

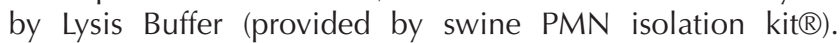
Lastly, polymorphonuclear neutrophils were washed with $10 \mathrm{~mL}$ cleaning solution (provided by swine PMN isolation $\mathrm{kit}(\mathbb{B})$ for three times and centrifuged at $250 \mathrm{~g}$ for $20 \mathrm{~min}$. All experiments were approved by the Ethics Committee on the Care and Use of Laboratory Animals of Foshan University and Jilin University.

\section{Isolation of spermatozoa}

The samples of seminal fluid $(n=6)$ were obtained from commercial semen used for breeding with normal semen parameters. The odor and color of seminal fluid are normal, and the sperm motility was above $80 \%$ at room temperature. For isolation of swine spermatozoa, seminal fluid was centrifugated at $650 \mathrm{~g}$ for $20 \mathrm{~min}$ and the sediment (spermatozoa) was washed three times and collected for next experiments.

\section{Scanning electron microscopy (SEM)}

Swine PMN were co-cultured with swine spermatozoa (ratio $1: 1$ ) cultured in phenol red-free RPMI media at $37^{\circ} \mathrm{C}$ for $2 \mathrm{~h}$. After co-incubation, the samples were fixed with $4.0 \%$ glutaraldehyde and treated as previous researches described (Wei et al. 2016, 2018a). In brief, samples were post-fixed in $1.0 \%$ osmium tetroxide, dehydrated in an ascending alcohol, frozen in tertiary butyl alcohol and sputtered with gold. Then, the specimens were observed and examined with a scanning electron microscope (Hitachi S-3400 N).

\section{Transmission electron microscopy (TEM)}

Swine PMN were co-cultured with swine spermatozoa (ratio 1:1) in phenol red-free RPMI media at $37^{\circ} \mathrm{C}$ for $2 \mathrm{~h}$. After co-incubation, the samples were fixed with $2.5 \%$ glutaraldehyde at $4{ }^{\circ} \mathrm{C}$ for $12 \mathrm{~h}$ and post-fixed with $1 \%$ osmium tetroxide for $1 \mathrm{~h}$. After that, the specimens were dehydrated in an ascending ethanol, infiltrated with spurr's resin, sliced into ultra-thin slices and stained with $4 \%$ uranyl acetate and $0.2 \%$ lead citrate. Subsequently, the specimens were observed and examined using transmission electron microscopy (Hitachi).

\section{Fluorescence confocal microscopy analyses}

Swine PMN were co-cultured with swine spermatozoa (ratio $1: 1)$ in phenol red-free RPMI media at $37^{\circ} \mathrm{C}$ for $2 \mathrm{~h}$ on polyL-lysine $(0.1 \mathrm{mg} / \mathrm{mL}$, Sigma-Aldrich) -treated coverslips. After fixed with $4 \%(\mathrm{w} / \mathrm{v})$ paraformaldehyde for $20 \mathrm{~min}$, the samples were permeabilized $(0.1 \%$ Triton X-100, $15 \mathrm{~min})$, blocked ( $3 \%$ BSA, 2 h) and incubated using specific antibodies (at $4{ }^{\circ} \mathrm{C}$ overnight). Five micrometer Sytox Orange $($ (Invitrogen) was used for staining NETs structures. Antibodies of anticitH3 (Citrulline R2 + R8 + R17; Ab5103; Abcam) or anti-MPO (Orb16003; Biorbyt) were used for detection of citH3 or MPO decorated with NETs. Finally, the images of NETs were observed by using scanning confocal microscope (Olympus FluoView FV1000).

\section{NETs quantitation}

The quantitation of NETs induced by swine spermatozoa (ratio $1: 1,1: 3$ or $1: 6$ ) in phenol red-free RPMI media at $37^{\circ} \mathrm{C}$ for 2 h was used by Pico Green ${ }^{\circledR}$ (Invitrogen). To further investigate the effect of seminal plasma on NETs formation, swine PMNs were exposed to sperm (ratio 1:1) or equivalent seminal plasma for $2 \mathrm{~h}$, and NETs formation was determined by Pico Green $^{\circledR}$ (Invitrogen) $(n=5)$. Zymosan ( $1 \mathrm{mg} / \mathrm{m} \mathrm{L}$; Sigma) used 

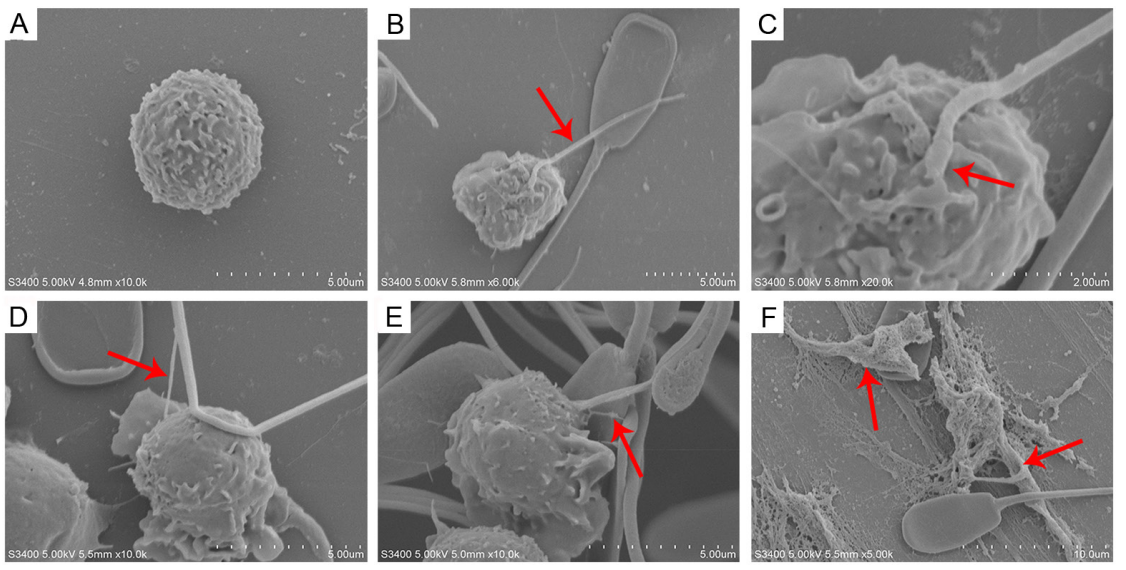

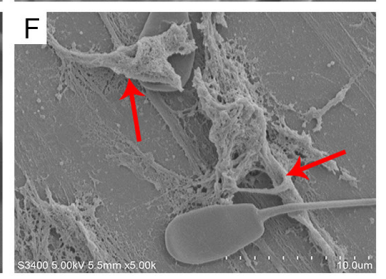

Figure 1 SEM analyses of swine spermtriggered NETs. Swine PMNs were exposed to sperm (ratio 1:1) for $2 \mathrm{~h}$ and the release of NETs was observed by SEM. (A) PMNs $(10.0 \mathrm{kX})$. (B) Heads of sperm captured by NETs (6.0 kX). (C) Amplification of (B) (20.0 kX). (D) Tails of sperm captured by thinner NETs-like structures (10.0 kX). (E) Tails of sperm captured by thinner NETs-like structures $(10.0 \mathrm{kX})$. (F) Sperm captured thicker NETs-like structures (5.0 kX). Arrow shows thinner or thicker NETs-like structures. Three separate experiments of SEM analysis were carried out to observe the structures of sperm-triggered NETs. as positive control as previously described (Wei et al. 2018a, Wei et al. 2016). In parallel groups, inhibitors of the NADPH oxidase (DPI, $10 \mu \mathrm{M}$, Sigma-Aldrich), ERK1/2 (UO126, 50 $\mu \mathrm{M}$, Sigma-Aldrich) or P38 MAPK (SB202190, $10 \mu \mathrm{M}$, SigmaAldrich) -signaling pathway were pretreated with swine PMN for $30 \mathrm{~min}$ and then stimulated with swine spermatozoa (ratio $1: 1)$ at $37^{\circ} \mathrm{C}$ for $2 \mathrm{~h}$. Furthermore, DNase I (90 U) was used to confirm the DNA nature of NETs. Afterward, the specimens were determined by Pico Green ${ }^{\circledR}$ and fluorometric reader Infiniti M200® (TECAN, Austria) as previously described (Wei et al. 2018b,c).

\section{Sperm motility analysis}

Sperm motility was examined through its activity and station of straight-line motion by using microscopic examination. Swine PMN were co-cultured with swine spermatozoa (ratio $1: 4,2: 4$ or $4: 4 ; 37^{\circ} \mathrm{C}$ ). The examination of sperm motility was performed at $0,1.5$ and $3 \mathrm{~h}$. Five random horizons of one specimen were selected to examine the sperm motility.

\section{In vitro fertilization and embryo culture}

Sperm and egg cells were separately obtained. By in vitro fertilization, diploid or tetraploid embryo was successfully acquired.

\section{Vital staining and fluorescence confocal microscopy analyses}

Swine PMN were co-cultured with diploid or tetraploid embryo (ratio $100: 1$ ) at $37^{\circ} \mathrm{C}$ for $1 \mathrm{~h}$ on confocal dish. During in vitro fertilization, several capacitating sperms always stick to the transparent tape of embryos, but can not be washed off embryos. While the embryo-PMN co-cultures, sperms also presented at the transparent tape of embryo and co-cultured with embryos. Then, Hoechst 33342 and Sytox Green (Invitrogen) were added for staining for $15 \mathrm{~min}$ in the culture solution. Hoechst 33342 is used to stain all of cells, but Sytox Green could just stain dead cells and NETs released but not living cells. Finally, the examination of the specimen was observed by using confocal microscope (Olympus FluoView FV1000).

\section{Statistical analysis}

Data were analyzed by the GraphPad 5.0 software and expressed as the means \pm s.D. One-way ANOVA with Tukey's multiple comparison tests were performed among the groups. Two-way ANOVA with Bonferroni post-tests were used for evaluate the difference of sperm motility. Significant difference among groups was regarded as a level of $P$-values $<0.05$.
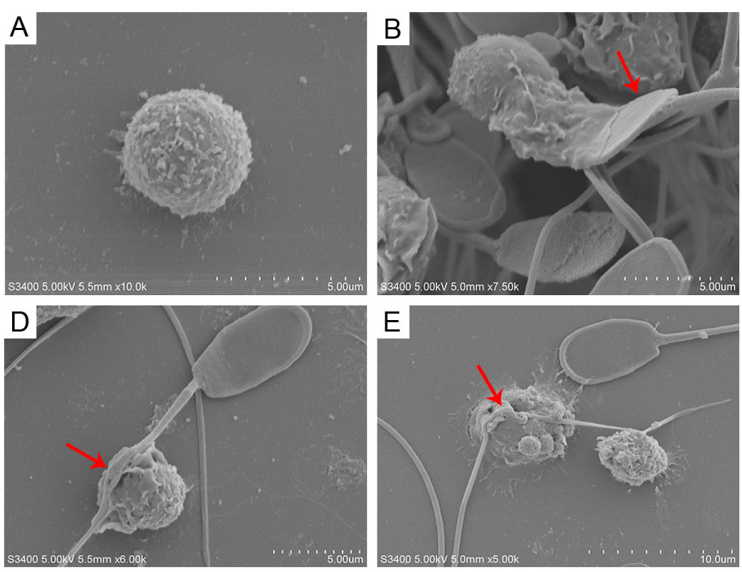
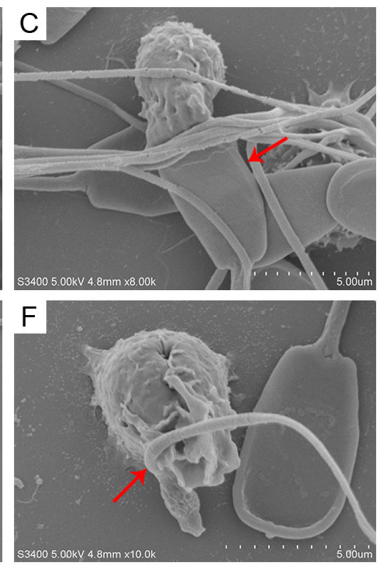

Figure 2 SEM analyses of swine sperm being engulfed or engulfed by PMNs. Swine PMNs were exposed to sperm (ratio 1:1) for $2 \mathrm{~h}$ and the phagocytosis of PMNs were observed by SEM. (A) PMNs (10.0 kX). (B) Heads of sperm being engulfed by PMNs (7.5 kX). (C) Heads of sperm being engulfed by PMNs (8.0 kX). (D) Tails of sperm captured being engulfed by PMNs $(6.0 \mathrm{kX})$. (F) Tails of sperm captured being engulfed by PMNs (5.0 kX). (E) Tails of sperm captured engulfed by PMNs (10.0 kX). Arrow shows sperm being engulfed or engulfed by PMNs. Three separate experiments of SEM analysis were carried out to observe sperm being engulfed or engulfed by PMNs. 

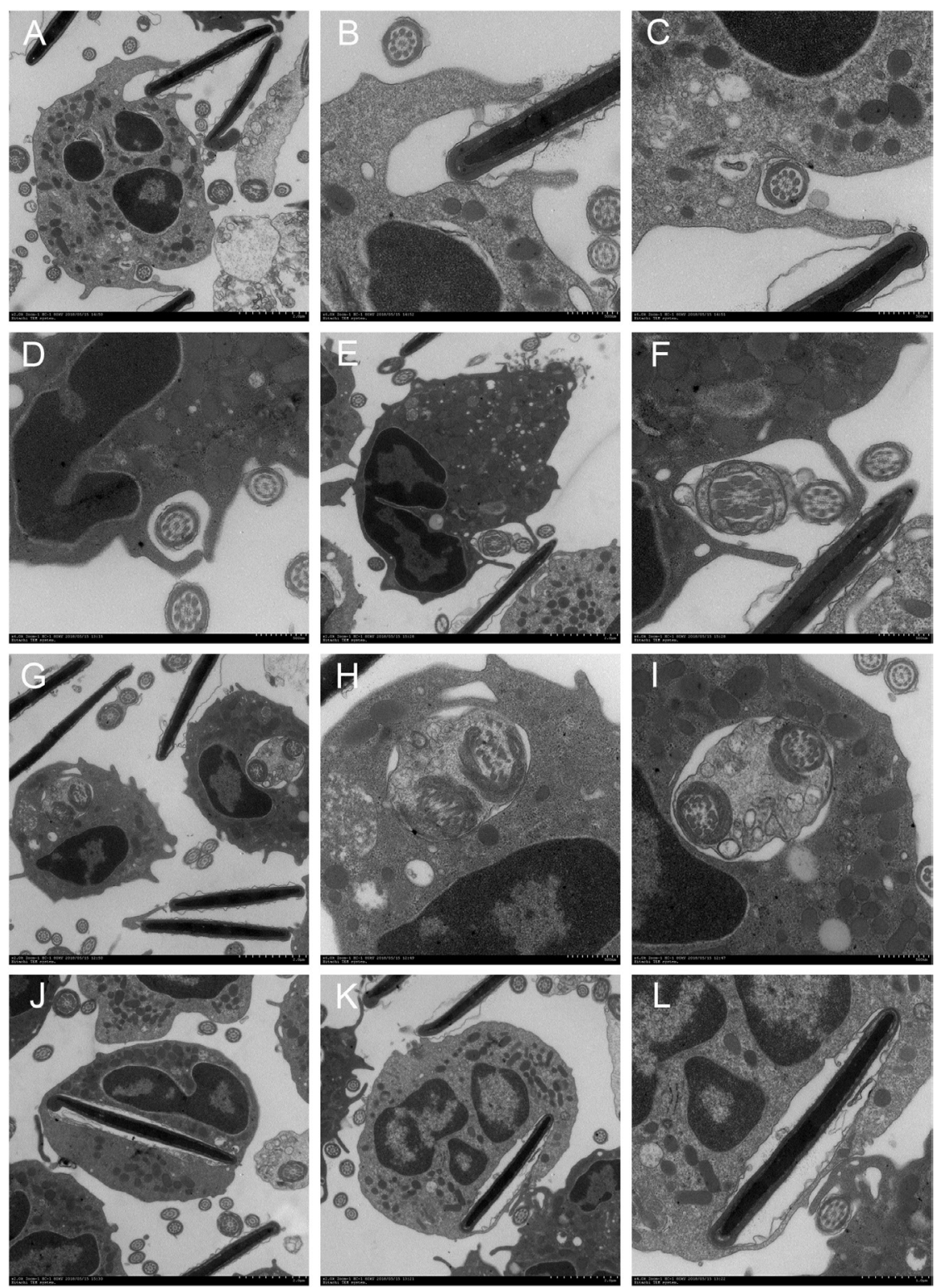

Figure 3 TEM analyses of swine sperm being engulfed or engulfed by PMNs. Swine PMNs were exposed to sperm (ratio 1:1) for $2 \mathrm{~h}$ and the phagocytosis of PMNs were observed by TEM. (A) Sperm being engulfed by PMNs. (B) Amplification of (A) showed heads of sperm being engulfed by PMNs. (C) Amplification of (A) showed tails of sperm being engulfed by PMNs. (D, E and F) Showed tails of sperm being engulfed by PMNs. (G, $\mathrm{H}$ and I) Showed tails of sperm engulfed by PMNs. (J, K and L) Heads of sperm engulfed by PMNs. Three separate experiments of TEM analysis were carried out to observe sperm being engulfed or engulfed by PMNs.

\section{Results}

\section{Swine sperm-triggered-NETs release}

Swine PMNs were exposed to sperm (ratio 1:1) for 2 $h$, and the release of NETs was first observed by SEM. The results showed that swine sperm triggered thickness of NETs-like structures ranging from thinner (Fig. 1B, $\mathrm{C}, \mathrm{D}$ and $\mathrm{E}$ ) to thicker (Fig. 1F). Moreover, not only the head (Fig. 1B) but also the tail (Fig. 1D) of the sperm was captured by these NETs-like structures.

\section{Phagocytosis occurred in the process of swine sperm -triggered-NETs release}

In the process of sperm-triggered-NETs release, swine sperm was also found to be engulfed by PMN phagocytosis. SEM analysis showed that the heads (Fig. 2B and C) or the tails (Fig. 2D and F) of sperm were being engulfed. Meanwhile, TEM analysis further revealed that the heads or tails of sperm were being engulfed (Fig. 3A, B, C, D, E and F), and the heads or tails of sperm were engulfed by PMNs (Fig. 3G, H, I, J, K and L).

\section{citH3 and MPO co-located with DNA in NETS structures triggered by swine sperm}

Swine PMNs were exposed to sperm (ratio 1:1) for $2 \mathrm{~h}$, and the release of NETs were also examined by immunofluorescence analysis. As shown in Fig. $4 \mathrm{~F}$ and $M$, swine sperm also triggered thinner or thicker NETslike structures release compared to PMN groups, and these structures were formed by citH3 (Fig. 4F) or MPO (Fig. 4M) co-located with DNA. 

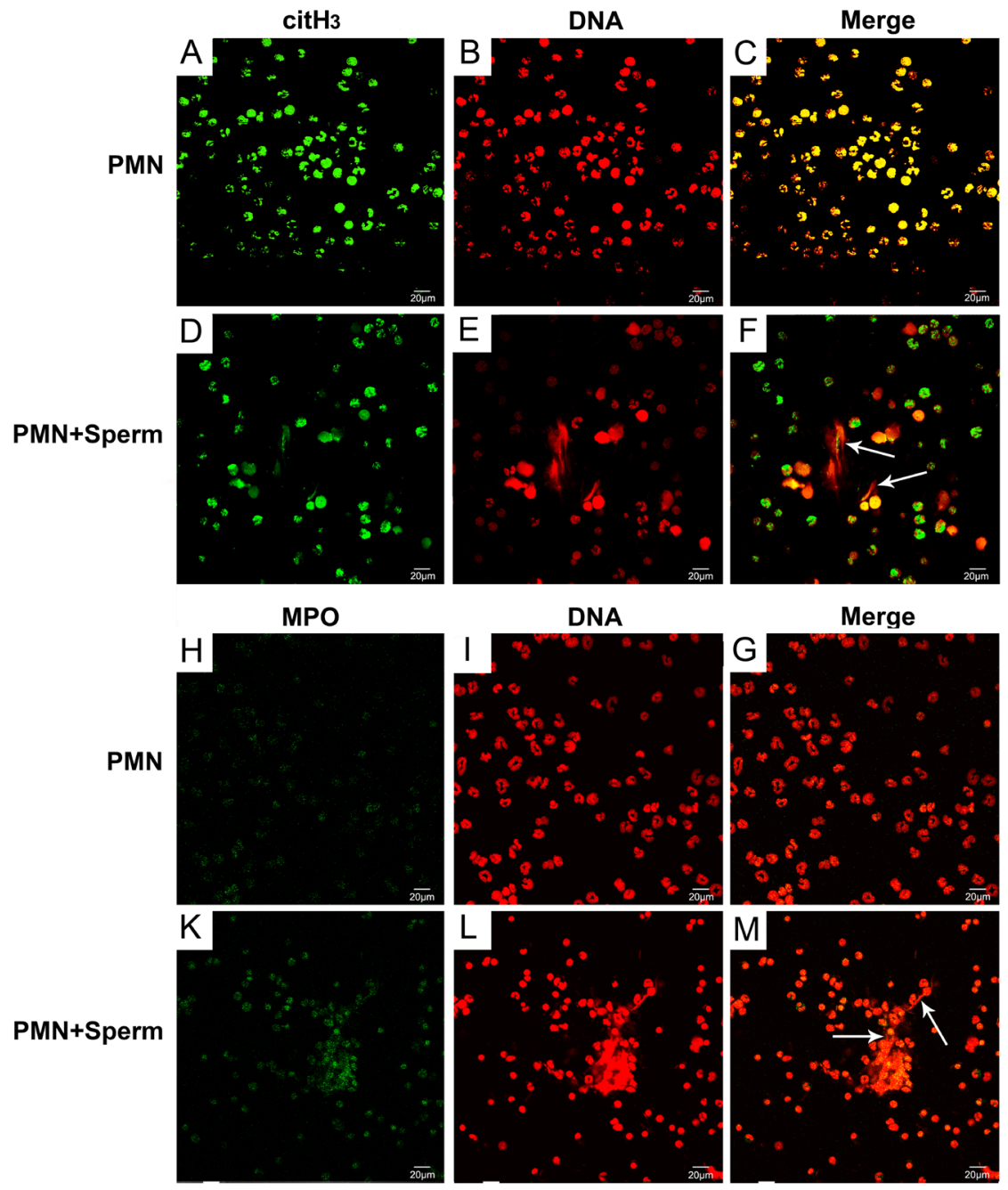

Figure 4 citH3 and MPO co-located with DNA in NETs triggered by swine sperm (400x). Swine PMNs were exposed to sperm (ratio $1: 1)$ for $2 \mathrm{~h}$ and the structures of NETs were examined by immunofluorescence analysis. (A) and (D) citH3 (Green). (B), (E), (I) and (L) DNA (Red). (H) and (K) MPO (Green). (C) Merge of A and B. (F) Merge of D and E. (G) Merge of $\mathrm{H}$ and I. (M) Merge of $\mathrm{K}$ and L. White arrows showed sperm-triggered NETs. Three separate experiments of immunofluorescence analysis were carried out to observe citH3 and MPO co-located with DNA in spermtriggered NETs.

\section{Quantitation of NETs triggered by swine sperm}

Quantitation of NETs formation showed that NETs formation in zymosan (positive control) has increased nearly four-fold compared to PMN groups and that swine sperm also significantly triggered NETs release in a dose-dependent manner (Fig. 5A), which confirmed the results of SEM analysis and immunofluorescence analysis that sperm did trigger NETs formation.

To further investigate whether seminal plasma could influence NETs formation, PMNs were exposed to sperm (ratio 1:1) or equivalent seminal plasma, and NETs formation was determined by Pico Green ${ }^{\circledR}$ (Invitrogen). The results showed that swine sperm significantly triggered nearly three-fold of NETs release compared with the control group, but seminal plasma did not significantly affect NETs formation (Fig. 5B).

\section{NADPH-, ERK1/2 and p38 signaling pathways involved in swine sperm-triggered-NETs release}

In parallel experiments, swine PMNs were pretreated with inhibitors of $\mathrm{NADPH}-$, ERK or p38 signaling pathways and then exposed to sperm. Quantitation of NETs showed that U0126, SB202190 or DPI significantly decreased NETs release triggered by sperm (Fig. 6). Furthermore, the release of NETs induced by swine sperm was also degraded by DNase I (Fig. 6), which also confirmed the main structure of DNA in NETs.

\section{Motility assay}

Swine PMNs were exposed to sperm (ratio 1:4, 2:4 or 4:4) and sperm motility was determined under microscopic examination at $0,1.5$ and $3 \mathrm{~h}$. As shown in Fig. 7, the sperm motility was slightly changed at low dose of PMNs (no significant difference; ratio 1:4), but it was significantly decreased at high dose PMNs exposed with sperm (ratio 2:4 or 4:4) compared to sperm group.

\section{Embryo entangled in the release of NETs}

By in vitro fertilization, diploid or tetraploid embryos of swine were acquired successfully (Fig. 8). After 2 h of 


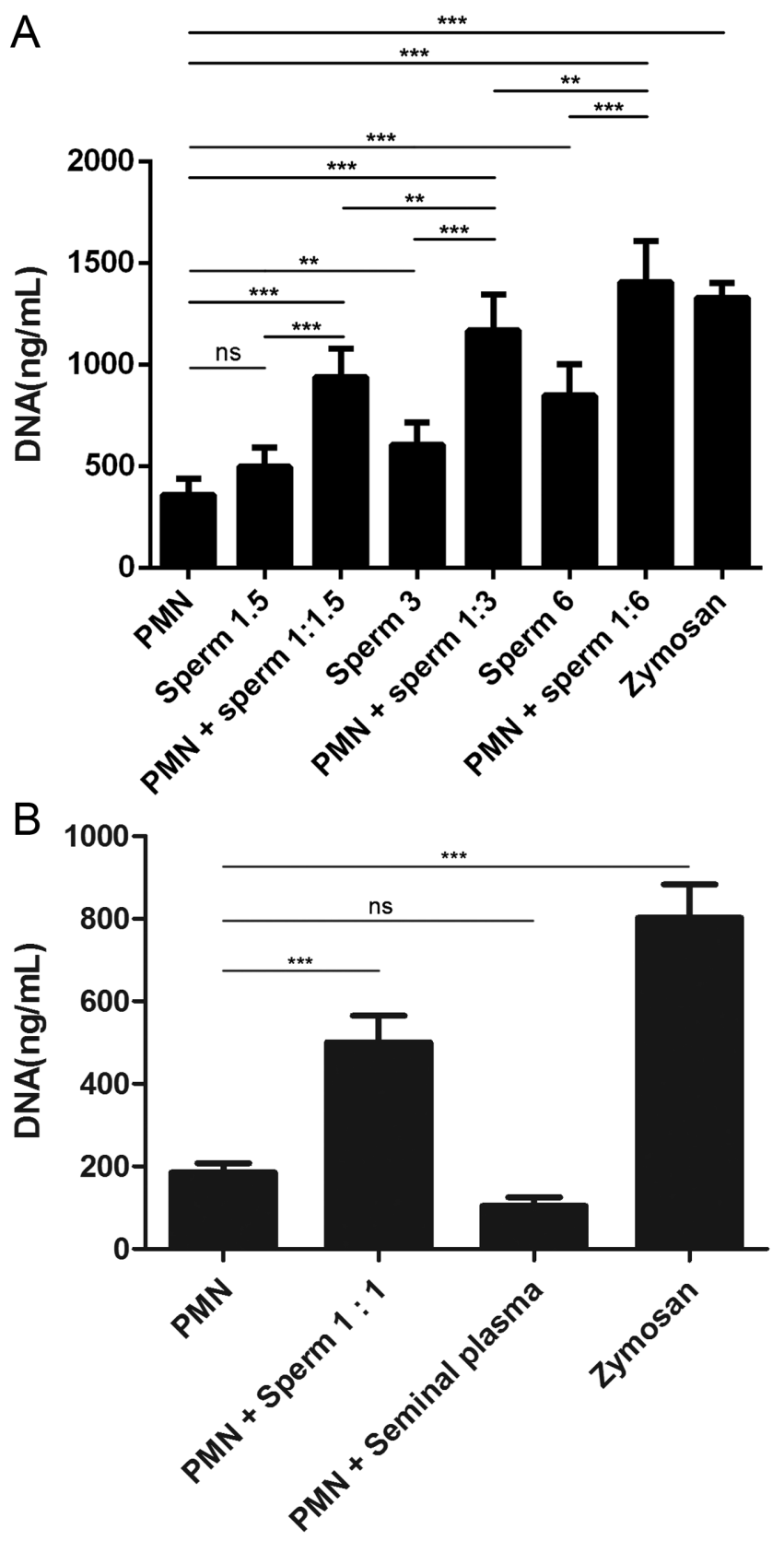

Figure 5 Quantitation of NETs triggered swine sperm. (A) Sperm triggered NETs formation in a dose-dependent manner. Swine PMNs were exposed to sperm (ratio 1:1.5, 1:3 or 1:6) for $2 \mathrm{~h}$ and NETs formation was determined by Pico Green ${ }^{\circledR}$ (Invitrogen) $(n=5)$. (B) Seminal plasma did not significantly affect NETs formation. Swine PMNs were exposed to sperm (ratio 1:1) or equivalent seminal plasma for $2 \mathrm{~h}$ and NETs formation was determined by Pico Green ${ }^{\circledR}$ (Invitrogen) $(n=5)$. Zymosan $(1 \mathrm{mg} / \mathrm{mL})$ was used in positive groups. Five separate experiments of quantitative NETs analysis were carried out to determine the effects of sperm on NETs formation. The data were presented as mean \pm S.D. $P$ values of $<0.05$ were considered significant. $\left({ }^{*} P<0.05\right.$, ${ }^{* *} P<0.01,{ }^{* * *} P<0.001$ and ' $n s^{\prime}$ means not significant).

co-culture, PMNs were congregated around embryos (Fig. 9A and K). Vital observation showed that PMNs were stained with Hoechst (Blue; Fig. 9B, G and L), but

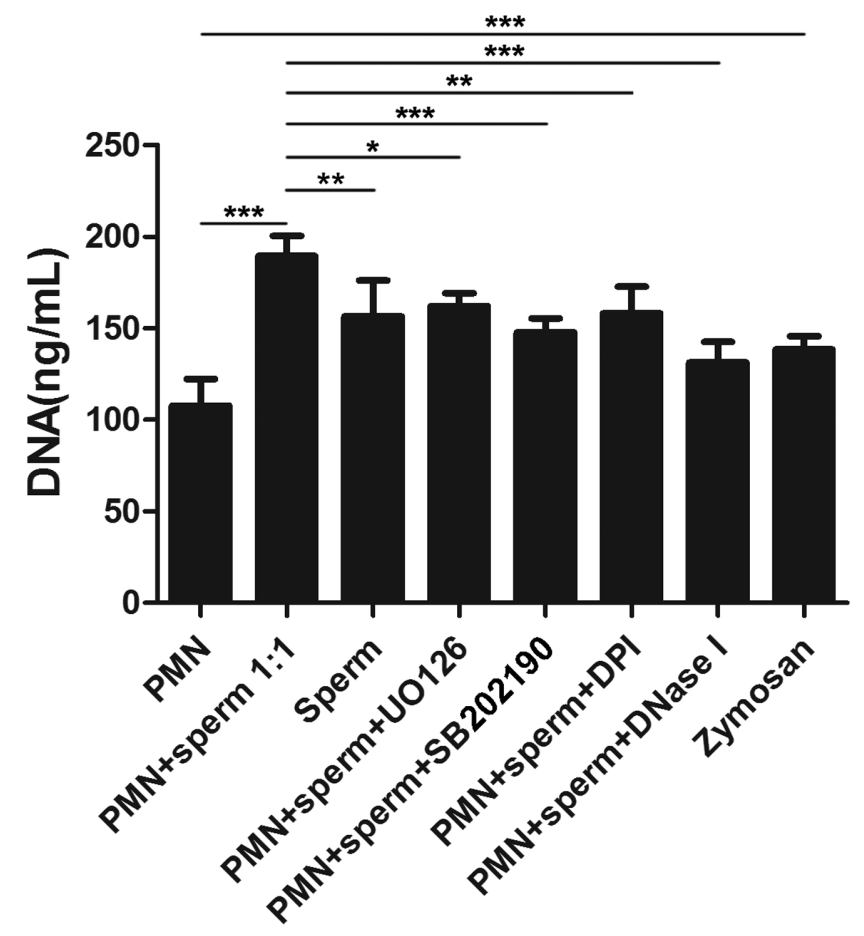

Figure 6 Effects of inhibitors of NADPH-, ERK1/2 or P38 MAPKsignaling pathway on NETs release. Inhibitors of NADPH-, ERK1/2 or P38 MAPK-signaling pathway were pretreated with swine PMNs. Then the swine PMNs were exposed to sperm (ratio 1:1) for $2 \mathrm{~h}$ and NETs formation was determined by Pico Green ${ }^{\circledR}$ (Invitrogen) $(n=5)$. Five separate experiments were carried out to determine the effects of inhibitors on sperm-triggered-NETs formation. The data were presented as mean \pm S.D. $P$ values of $<0.05$ were considered significant. $\left({ }^{*} P<0.05,{ }^{* *} P<0.01,{ }^{* * *} P<0.001\right.$ and 'ns' means not significant).

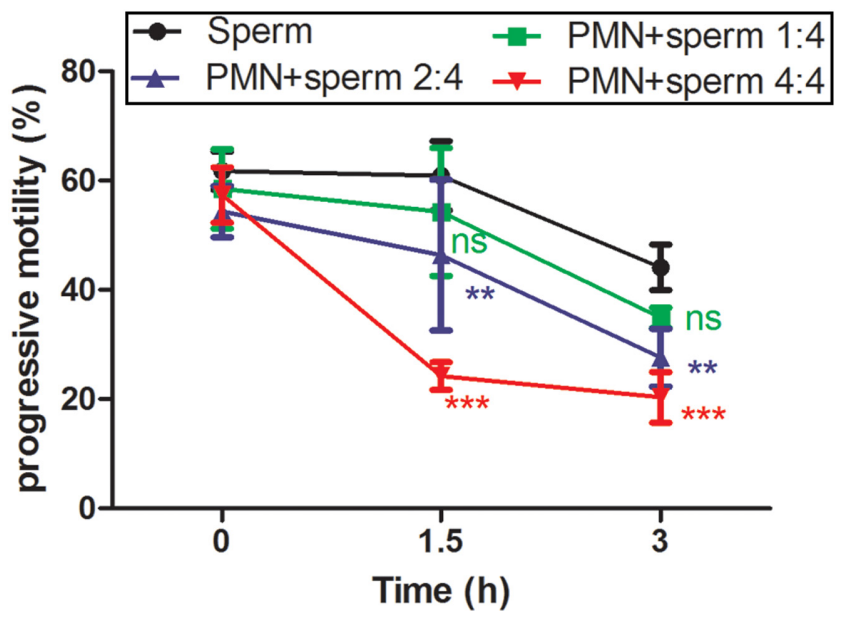

Figure 7 Effects of swine PMNs on sperm motility. Swine PMN were co-cultured with swine spermatozoa (ratio $1: 4,2: 4$ or $4: 4 ; 37^{\circ} \mathrm{C}$ ), and the examination of sperm motility was performed at $0,1.5$ and $3 \mathrm{~h}$ $(n=3)$. Three separate experiments were carried out to determine the effects of swine PMNs on sperm motility. The data were presented as mean \pm S.D. $P$ values of $<0.05$ were considered significant. (**P $<0.01,{ }^{* * *} P<0.001$ and 'ns' means not significant). 

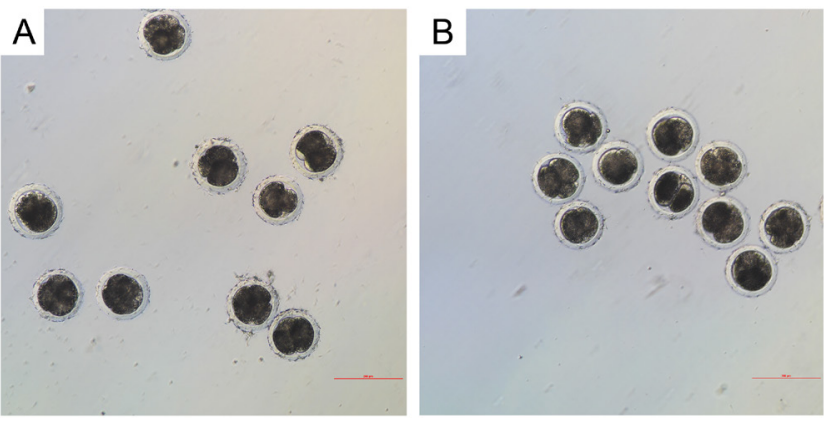

Figure 8 Optical images of diploid or tetraploid embryo. Diploid or tetraploid embryo was successfully acquired by in vitro fertilization. Then, optical images were collected $(200 \mu \mathrm{m})$.

only dead PMNs, head of sperm on embryo and NETs were stained with Sytox Green (Green; Fig. 9C, H and $\mathrm{M})$. More interestingly, embryo was entangled in the release of NETs (Fig. 9E and O).

\section{Discussion}

The presented data confirmed that PMNs were directly activated in forms of phagocytosis or casting NETs by swine sperm, like the phenomenon observed in other species such as human (Zambrano et al. 2016), bovine (Marey et al. 2014) or horse (Alghamdi \& Foster 2005). There is no species difference from other species reported in this respect. In addition, there is also no site specific of sperm captured by these thinner or thicker of NETs-like structures (Fig. 1). More interestingly, PMN phagocytosis of sperm was also found in the same time point of NETs release (Fig. 2). To further confirm the phagocytosis of MPNs on swine sperm, TEM analysis was carried out. The result showed that the heads or tails of sperm were being engulfed or engulfed by PMNs (Fig. 3), which suggested that phagocytosis and NETs work together to eliminate excess sperm in normal physiological conditions. Of note is that this synergistic activity may also result in infertility. However, the occurrence of phagocytosis and NETs release in a single PMN has not been observed.

In this study, SEM analysis was used to observe the morphology of sperm-induced NETs which revealed the thinner or thicker of NETs-like structures triggered by swine sperm. It is reported that typical NETs structures are made up of DNA decorated with histone, MPO or other proteins (Brinkmann et al. 2004, Munoz-Caro et al. 2015b, Wei et al. 2018a). Whether these NETs-like structures triggered by swine sperm possess the typical characterize of NETs remain unclear. Thus, fluorescence confocal microscopy analysis was used to prove the
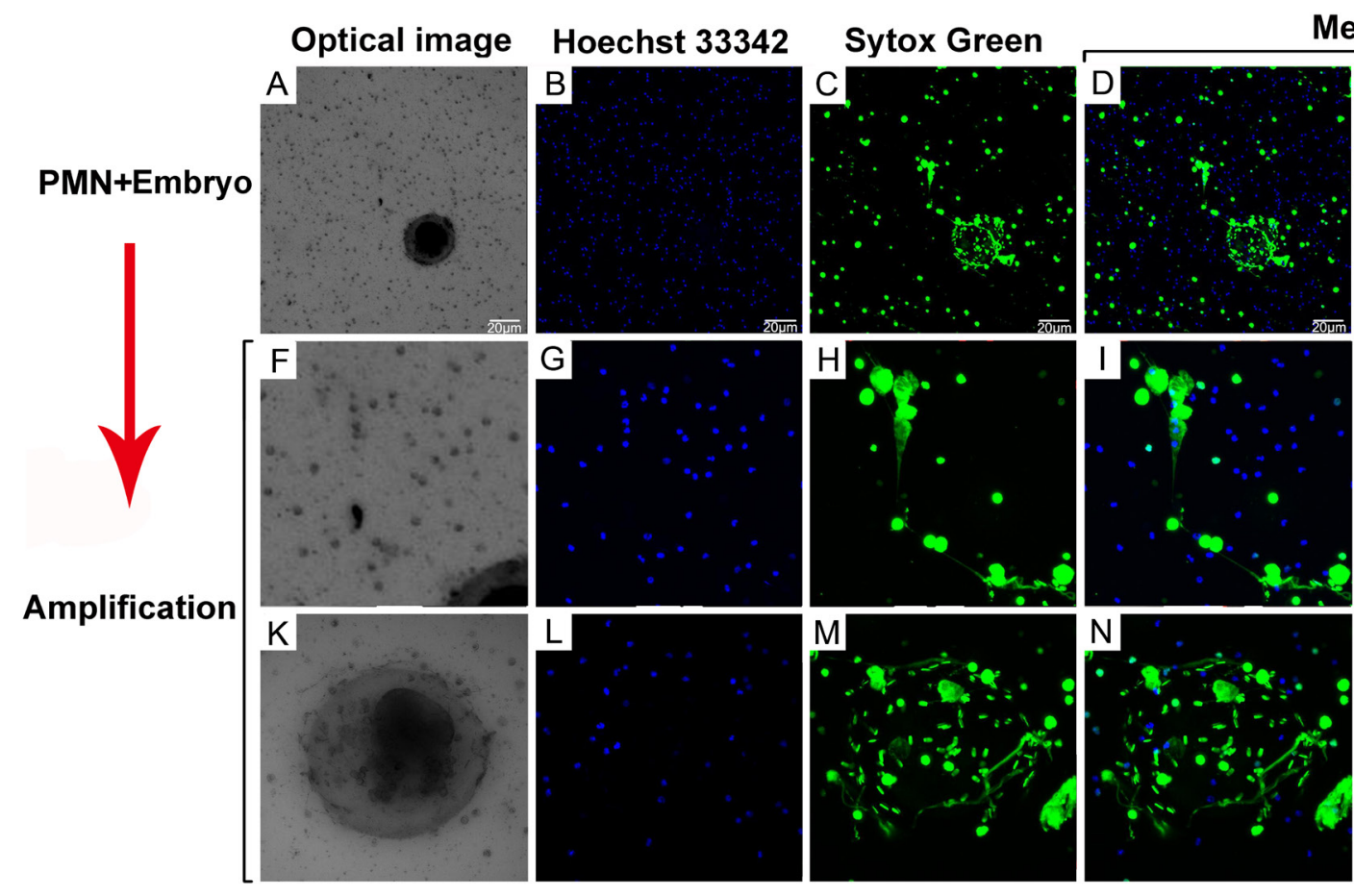

Merge
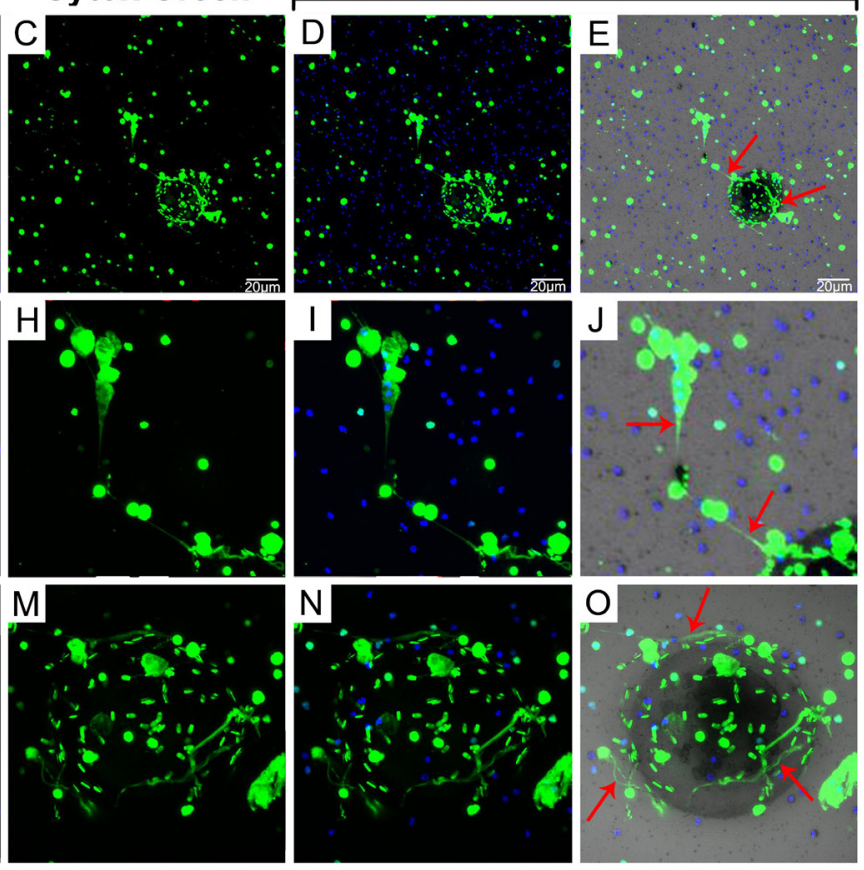

Figure 9 Vital staining of embryo entangled in the release of NETs. Swine PMNs were co-cultured with diploid or tetraploid embryo (ratio 100:1) at $37^{\circ} \mathrm{C}$ for $1 \mathrm{~h}(n=3)$. While embryo-PMN co-culturesyes, sperms also presented at the transparent tape of embryo and co-cultured with embryos. Then the species were observed by scanning confocal microscope (400X). (A), (F) and (K) Optical image showed PMNs were congregated around embryos. (B), (G) and (L) Hoechst33342 (Blue). (C), (H) and (M) Sytox Green (Green). (D) Merge of B and (C). (I) Merge of G and $\mathrm{H}$. (N) Merge of $\mathrm{L}$ and M. Red arrows showed NETs around embryo. Three separate experiments were carried out to observe embryo entangled in the release of NETs. 
typical structures of sperm-triggered NETs. It has been found that sperm significantly triggered NETs release and that these structures were composed of DNA decorated with citH3 and MPO (Fig. 4), which revealed the typical NETs-like structures triggered by sperm. In addition, the quantitative method of NETs analysis was further used to detect sperm-induced NETs release, and the results showed that swine sperm could significantly trigger NETs release in a dose-dependent manner. All these results confirm that swine sperm do activate PMNs casting NETs, which were similar to researches on human sperm (Zambrano et al. 2016), bacteria (Johnson et al. 2016), virus (Saitoh et al. 2012) or parasites (Reichel et al. 2015, Wei et al. 2018a). However, whether other factors such as cytokines, chemokines or others secreted by sperm-stimulated PMNs caused NETs formation remain unknown and need to be clarified in the future research.

Mechanisms involved in sperm-triggered-NETs release remain unknown, thus we perform the quantitative analysis of NETs release with several inhibitors. First, we detected if swine sperm-NETs formation depends on NADPH oxidase, and the results showed that the inhibitor of NADPH oxidase (DPI) decreased sperminduced NETs formation in a similar degree among human, bovine and swine, which meant that all different kinds of sperm (human, bovine and swine) -induced NETs formation were NOX-dependent processes. It has been also showed that sperm-triggered-NETs formation was also dependent on ERK1/2 or p38 MAPK signaling pathway. Moreover, the release of sperm-triggered NETs was degraded by DNase I, which further confirmed the properties of DNA in the main components of NETs. Of further note is that seminal plasma improves the fertility in equine and swine sperm (Rozeboom et al. 2000, Alghamdi et al. 2004) and that seminal DNase also help spermatozoa escape from NETs (Alghamdi \& Foster 2005). Our results further showed that seminal plasma could decrease NETs formation spontaneously in PMNs, but its distinction did not prove a statistical significance (Fig. 5B). During the normal process of fertilization, seminal fluid could prevent excess NETs release. Whether the changes of $\mathrm{pH}$ in the ejaculate or female vagina would be protective against NET release still need to be further investigated. Investigation of sperm motility analysis demonstrated that sperm motility was significantly reduced as more PMNs added in the experimental system. Previously mentioned results may help explain the reduced fertility occuring in overdiluted semen inseminations or repeated inseminations leading to reproductive tract leukocytosis.

Now that the NETs release or phagocytosis of swine PMNs has a negative influence on sperm motility, but whether embryo implantation also affected by $\mathrm{PMN}$ s remains unclear. By in vitro fertilization, we have acquired diploid or tetraploid embryos of swine, and further vital staining analyses revealed that sperm adhered to the embryo also could trigger NETs that entangled the diploid or tetraploid embryos (Fig. 9). Of great interest is whether the embryo entangled in NETs is good for implantation in vivo or not and still needs further investigation.

Taken together, this study shows that phagocytosis and NETs of PMNs work together to reduce sperm motility. Especially, NETs could also capture embryo in vitro, but the function and mechanism of this action in vivo fertilization is not yet known.

\section{Declaration of interest}

The authors declare that there is no conflict of interest that could be perceived as prejudicing the impartiality of the research reported.

\section{Funding}

This work was funded by the National Natural Science Foundation of China (No. 31772721).

\section{Author contribution statement}

Zhengtao Yang, Hongsheng Ouyang and Zhengkai Wei designed the project and experiments. Zhengkai Wei, Tingting Yu, Chaoqun Wang, Xiao Liu, Zhen Han, Jingjing Wang, Xu Zhang and Yong Zhang carried out the experiments. Zhengkai Wei wrote the manuscript and carried out statistical analysis and prepared figures. All authors reviewed the manuscript.

\section{References}

Agraz-Cibrian JM, Giraldo DM, Mary FM \& Urcuqui-Inchima S 2017 Understanding the molecular mechanisms of NETs and their role in antiviral innate immunity. Virus Research 228 124-133. (https://doi. org/10.1016/j.virusres.2016.11.033)

Alghamdi AS \& Foster DN 2005 Seminal DNase frees spermatozoa entangled in neutrophil extracellular traps. Biology of Reproduction 73 1174-1181. (https://doi.org/10.1095/biolreprod.105.045666)

Alghamdi AS, Foster DN \& Troedsson MH 2004 Equine seminal plasma reduces sperm binding to polymorphonuclear neutrophils (PMNs) and improves the fertility of fresh semen inseminated into inflamed uteri. Reproduction 127 593-600. (https://doi.org/10.1530/rep.1.00096)

Alghamdi AS, Lovaas BJ, Bird SL, Lamb GC, Rendahl AK, Taube PC \& Foster DN 2009 Species-specific interaction of seminal plasma on sperm-neutrophil binding. Animal Reproduction Science 114 331-344. (https://doi.org/10.1016/j.anireprosci.2008.10.015)

Brinkmann V, Reichard U, Goosmann C, Fauler B, Uhlemann Y, Weiss DS, Weinrauch Y \& Zychlinsky A 2004 Neutrophil extracellular traps kill bacteria. Science 303 1532-1535. (https://doi.org/10.1126/ science.1092385)

Douda DN, Khan MA, Grasemann H \& Palaniyar N 2015 SK3 channel and mitochondrial ROS mediate NADPH oxidase-independent NETosis induced by calcium influx. PNAS 112 2817-2822. (https://doi. org/10.1073/pnas.1414055112)

Eisenbach M 2003 Why are sperm cells phagocytosed by leukocytes in the female genital tract? Medical Hypotheses 60 590-592. (https://doi. org/10.1016/s0306-9877(03)00054-9)

Fichtner T, Kotarski F, Gartner U, Conejeros I, Hermosilla C, Wrenzycki C \& Taubert A 2020 Bovine sperm samples induce different NET phenotypes in a NADPH oxidase-, PAD4- and Ca++-dependent process. Biology of Reproduction 102 902-914. (https://doi.org/10.1093/biolre/ioaa003) 
Hahn S, Giaglis S, Hoesli I \& Hasler P 2012 Neutrophil NETs in reproduction: from infertility to preeclampsia and the possibility of fetal loss. Frontiers in Immunology 3 362. (https://doi.org/10.3389/ fimmu.2012.00362)

Han Z, Zhang Y, Wang C, Liu X, Jiang A, Liu Z, Wang J, Yang Z \& Wei Z 2019 Ochratoxin A-triggered chicken heterophil extracellular traps release through reactive oxygen species production dependent on activation of NADPH oxidase, ERK, and p38 MAPK signaling pathways. Journal of Agricultural and Food Chemistry 67 11230-11235. (https:// doi.org/10.1021/acs.jafc.9b03155)

Jenne CN, Wong CH, Zemp FJ, Mcdonald B, Rahman MM, Forsyth PA, Mcfadden G \& Kubes P 2013 Neutrophils recruited to sites of infection protect from virus challenge by releasing neutrophil extracellular traps. Cell Host and Microbe 13 169-180. (https://doi.org/10.1016/j. chom.2013.01.005)

Johnson CJ, Cabezas-Olcoz J, Kernien JF, Wang SX, Beebe DJ, Huttenlocher A, Ansari H \& Nett JE 2016 The extracellular matrix of Candida albicans biofilms impairs formation of neutrophil extracellular traps. PLoS Pathogens 12 e1005884. (https://doi.org/10.1371/journal. ppat.1005884)

Kaeoket K, Persson E \& Dalin AM 2003 Influence of pre-ovulatory insemination and early pregnancy on the infiltration by cells of the immune system in the sow endometrium. Animal Reproduction Science 75 55-71. (https://doi.org/10.1016/s0378-4320(02)00230-0)

Katila T 2012 Post-mating inflammatory responses of the uterus. Reproduction in Domestic Animals 47 (Supplement 5) 31-41. (https:// doi.org/10.1111/j.1439-0531.2012.02120.x)

Knox RV 2016 Artificial insemination in pigs today. Theriogenology 85 83-93. (https://doi.org/10.1016/j.theriogenology.2015.07.009)

Marey MA, Liu J, Kowsar R, Haneda S, Matsui M, Sasaki M, Takashi S, Hayakawa H, Wijayagunawardane MP, Hussein FM et al. 2014 Bovine oviduct epithelial cells downregulate phagocytosis of sperm by neutrophils: prostaglandin E2 as a major physiological regulator. Reproduction 147 211-219. (https://doi.org/10.1530/REP-13-0375)

Munoz-Caro T, Huertas SJM, Conejeros I, Alarcon P, Hidalgo MA, Burgos RA, Hermosilla C \& Taubert A 2015a Eimeria bovis-triggered neutrophil extracellular trap formation is CD11b-, ERK 1/2-, p38 MAP kinase- and SOCE-dependent. Veterinary Research 46 23. (https://doi org/10.1186/s13567-015-0155-6)

Munoz-Caro T, Lendner M, Daugschies A, Hermosilla C \& Taubert A 2015b NADPH oxidase, MPO, NE, ERK1/2, p38 MAPK and Ca2+ influx are essential for Cryptosporidium parvum-induced NET formation. Developmental and Comparative Immunology 52 245-254. (https://doi. org/10.1016/j.dci.2015.05.007)

Reichel M, Munoz-Caro T, Sanchez Contreras G, Rubio Garcia A, Magdowski G, Gartner U, Taubert A \& Hermosilla C 2015 Harbour seal (Phoca vitulina) PMN and monocytes release extracellular traps to capture the apicomplexan parasite Toxoplasma gondii. Developmental and Comparative Immunology 50 106-115. (https://doi.org/10.1016/j. dci.2015.02.002)

Rozeboom KJ, Troedsson MH \& Crabo BG 1998 Characterization of uterine leukocyte infiltration in gilts after artificial insemination. Journal of Reproduction and Fertility 114 195-199. (https://doi.org/10.1530/ jrf.0.1140195)

Rozeboom KJ, Troedsson MH, Hodson HH, Shurson GC \& Crabo BG 2000 The importance of seminal plasma on the fertility of subsequent artificial inseminations in swine. Journal of Animal Science 78 443-448. (https:// doi.org/10.2527/2000.782443x)

Saitoh T, Komano J, Saitoh Y, Misawa T, Takahama M, Kozaki T, Uehata T, Iwasaki H, Omori H, Yamaoka S et al. 2012 Neutrophil extracellular traps mediate a host defense response to human immunodeficiency virus-1. Cell Host and Microbe 12 109-116. (https://doi.org/10.1016/j. chom.2012.05.015)

Wei Z, Hermosilla C, Taubert A, He X, Wang X, Gong P, Li J, Yang Z \& Zhang X 2016 Canine neutrophil extracellular traps release induced by the apicomplexan parasite Neospora caninum in vitro. Frontiers in Immunology 7 436. (https://doi.org/10.3389/fimmu.2016.00436)

Wei Z, Wang Y, Zhang X, Wang X, Gong P, Li J, Taubert A, Hermosilla C, Zhang X \& Yang Z 2018a Bovine macrophage-derived extracellular traps act as early effectors against the abortive parasite Neospora caninum. Veterinary Parasitology 258 1-7. (https://doi.org/10.1016/j. vetpar.2018.06.002)

Wei Z, Zhang X, Wang J, Wang Y, Yang Z \& Fu Y 2018b The formation of canine neutrophil extracellular traps induced by sodium arsenic in polymorphonuclear neutrophils. Chemosphere 196 297-302. (https:// doi.org/10.1016/j.chemosphere.2017.12.175)

Wei Z, Zhang X, Wang Y, Wang J, Fu Y \& Yang Z 2018c Nickel (II) nitrate hexahydrate triggered canine neutrophil extracellular traps release in vitro. Chemosphere 208 117-121. (https://doi.org/10.1016/j. chemosphere.2018.05.184)

Wood GA, Fata JE, Watson KL \& Khokha R 2007 Circulating hormones and estrous stage predict cellular and stromal remodeling in murine uterus. Reproduction 133 1035-1044. (https://doi.org/10.1530/REP-06-0302)

Yang Z, Wei Z, Hermosilla C, Taubert A, He X, Wang X, Gong P, Li J \& Zhang X 2017 Caprine monocytes release extracellular traps against Neospora caninum in vitro. Frontiers in Immunology 8 2016. (https:// doi.org/10.3389/fimmu.2017.02016)

Yeste M, Rodriguez-Gil JE \& Bonet S 2017 Artificial insemination with frozen-thawed boar sperm. Molecular Reproduction and Development 84 802-813. (https://doi.org/10.1002/mrd.22840)

Zambrano F, Carrau T, Gartner U, Seipp A, Taubert A, Felmer R, Sanchez R \& Hermosilla C 2016 Leukocytes coincubated with human sperm trigger classic neutrophil extracellular traps formation, reducing sperm motility. Fertility and Sterility 106 1053.e1-1060.e1. (https://doi.org/10.1016/j. fertnstert.2016.06.005)

Received 19 July 2019

First decision 16 September 2019

Revised manuscript received 20 April 2020

Accepted 14 May 2020 